\title{
DCLK1 expression in gastrointestinal stem cells and neoplasia
}

Giuseppe Gagliardi ${ }^{1}$ and Charles F. Bellows ${ }^{1 *}$

Correspondence: cbellows@tulane.edu

${ }^{1}$ Tulane University, Department of Surgery SL-22, 1430 Tulane Ave, New Orleans LA 70112, USA.

\begin{abstract}
Doublecortin-like kinase (Dclk1), a gene involved in neurogenesis, has recently been found expressed in single cells of the human and murine adult gastrointestinal tract. Currently, there are opposing opinions on the exact identity and functional characteristics of DCLK1 positive cells. Some view them as gastrointestinal stem cells that fuel the self-renewal process, whereas others view them as an intestinal brush cells or as an enteroendocrine subtype. Interestingly, Dclk1 is over-expressed in several gastrointestinal malignancies, but is expressed at only low levels in normal gastrointestinal cells, making it a possible target for cancer therapy. In view of the recent therapeutic efforts to antagonize and target the cancer stem cell population, there has been ongoing interest in DCLK1. In this review we critically appraise the studies describing the expression of Dclk1 in the normal and neoplastic gastrointestinal tract as well as discuss the possible function and of Dclk1 in the gastrointestinal system and the potential clinical relevance as a tumor marker and a therapeutic target.
\end{abstract}

Keywords: Dclk1, Dcamkl-1, intestinal stem cells, cancer stem cells, colorectal neoplasms, pancreatic neoplasms, esophageal neoplasms, liver neoplasms, brush cells, Sox 9, SiRNA, tuft cells.

\section{Background}

The human doublecortin gene family includes 11 members that share the tubulin-binding domain and are involved in neural development [1]. One of the most interesting members of this family is doublecortin-like kinase 1 (Dclk1 also known as Dcamkl-1), a gene encoding for a protein that is $70 \%$ identical to doublecortin in the microtubule-binding $\mathrm{N}$-terminal domain. However, unlike doublecortin, the Dclk1 gene also encodes for a serine-threonine kinase $\mathrm{C}$-terminal domain that is similar to Ca2+/calmodulin-dependent protein kinase II, but lacks a canonical calmodulin-binding site. The Dclk1 gene is extremely complex, and has at least nine alternative splice variants, including a full length isoform with an arginine-rich domain, an isoform containing only the microtubule-binding domain and an isoform containing only the kinase domain, which are expressed differently in various areas of the brain [2]. The function of the DCLK1 protein in not well known but is thought to overlap with that of doublecortin. The tubulin binding part of the protein is involved in shaping the cytoskeleton, thereby regulating cell motility and axonal migration as well as differentiation and the cell cycle while the protein kinase function is unknown. In addition, sequence analysis has identified at least one transmembrane domain near the C-terminus and several phosphorylation sites, which suggest its involvement in signaling pathways [3].

The Dclk1 gene is mainly expressed in the fetal and adult brain, although lower levels of the transcripts are also found in the fetal lung and liver as well as the adult heart, liver, stomach, thymus, prostate, testis, small intestine and colon[4]. More recently, the Dclk1 gene was found to be highly expressed in the stem cell zone of mouse gastric glands and was proposed as a new gastrointestinal stem cell marker. In the past few years several studies have attempted to further define Dclk1 expression and function in the human and mouse gastrointestinal tract, often with conflicting results. The purpose of this review is to critically appraise the studies describing the expression of Dclk 1 in the normal and neoplastic gastrointestinal tract as well as discuss the function and potential clinical relevance of Dclk1 in the gastrointestinal system.

Early evidence of DCLK1 as a gastrointestinal stem cell marker DCLK1 has only recently been identified in the gastrointestinal tract. In 2006, Giannakis and colleagues showed increased Dclk1 expression in the isthmus of gastric glands, which is the presumed location of long lived, quiescent stem cells [5]. DCLK1 localization to the isthmus of gastric glands and to the +4 position of intestinal crypts was subsequently confirmed by immunohistochemical staining. It was also shown that DCLK1 cells did not incorporate the thymidine analogue bromo-deoxy uridine (BRDU), which is consistent with lack of cell proliferation typical of quiescent stem cells [5]. Moreover, DCLK1 positive cells did not co-localize with markers of differentiated gastric cells, which suggests that these cells may be undifferentiated progenitor cells [5].

Adult stem cells have the capacity of self renew and of replacing differentiated cells that are lost each day. A population of rapidly proliferating crypt-base stem cells that co-express Lgr5 maintain the fast turnover of the intestinal epithelium [6]. 


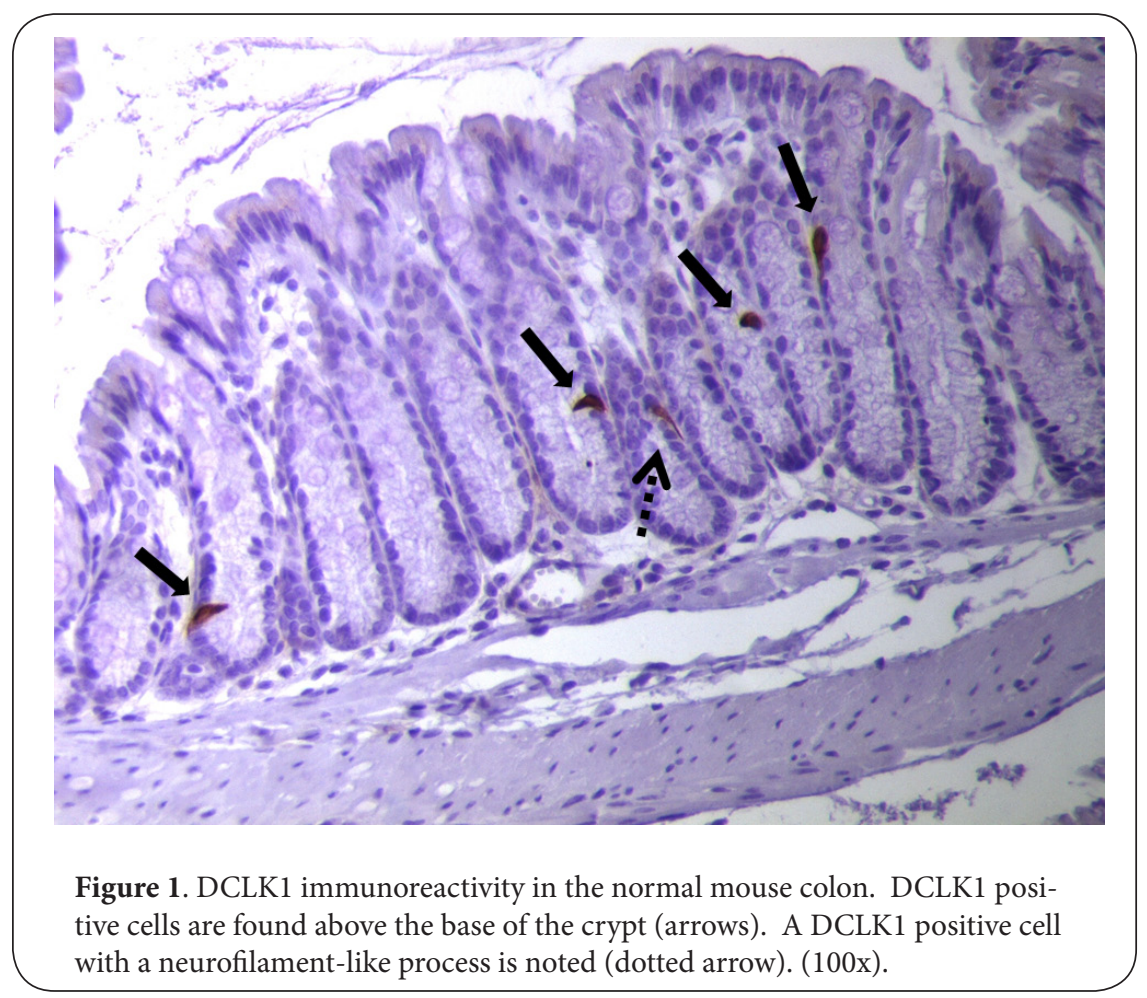

However, it has been postulated that the stem cells should divide infrequently in order to prevent the acquisition of errors during DNA replication and to preserve their long-term pluripotency. Early cell tracking experiments located this quiescent stem cell population in the +4 position in the small intestine and in the isthmus of the gastric glands [7]. These slowly cycling stem cells have only recently been found to exclusively express Bmi1 and Mtert $[7,8]$, but the characterization of these cells and their co-existence alongside, and perhaps upstream of the Lgr5 positive population remains controversial [7]. Therefore, the search continues for the identification of new markers for this elusive stem cell population.

In 2008, May and colleagues confirmed the finding that DCLK1 is mainly expressed in single cells in the +4 stem cell zone within the mouse small intestine and colonic crypts [9]. In this tissue, DCLK1 positive cells were noted to have a fusiform morphology with axonal like processes similar to gastric $D$ cells, a type of enteroendocrine cells that produces somatostatin (Figure 1). However, co-localization studies with the enteroendocrine markers $\mathrm{CgA}$, somatostatin and secretin appeared negative, suggesting that DCLK1 cells were different from mature enteroendocrine cells $[9,10]$. Further co-localization studies with known stem cell markers showed no co-localization with MSI-1 or LGR5, as well as with the proliferation marker PCNA, which was highly expressed in the LGR5 positive transient amplifying cell population[10]. Since DCLK1 cells were localized above the crypt base cells and lacked of co-localization with other stem cell markers, DCLK1 was thought to be a unique marker of quiescent stem cells.
Adding further evidence to the hypothesis that DCLK1 is a marker of intestinal stem cells, the response of DCLK1 positive cells to radiation was studied in a mouse model.

Regeneration of the crypt after radiation injury relies upon non-replicating stem cells. After 6 Gy of radiation DCLK1 cells resisted the first wave of apoptosis, considered to affect stem cells of the +4 compartment [6], but underwent apoptosis and mitosis after 24-48 hours[9]. However, after a sterilizing dose of radiation (12 Gy), DCLK1 cells did not take part in the proliferative phase, a process where the only transient amplifying progenitor cells are involved, and re-appeared at 7 days. To provide further evidence of DCLK1 cell quiescence, the authors performed a modified label retention assay after 8 Gy of radiation, which showed that DCLK1 cells were the only cells to retain label after 10 days [10].

More evidence in support of the stem cell nature of DCLK1 positive cells was provided by in vitro isolation studies. In low attachment culture, DCLK1 positive cells were able to grow and form spheres, a capacity attributed only to stem cells [10]. Furthermore, DCLK1 positive cells injected in nude mice were able to form gland like structures and give rise to all intestinal cell lineages, which was considered as proof of their pluripotency [10]. Taken together, these experiments lead scientist to the early believe that DCLK1 was a gastrointestinal stem cell marker and, in particular, a marker of the quiescent stem cells from the +4 compartment $[7,11-16]$. However, these findings were rapidly followed by several studies that dampened the scientific enthusiasm of DCKL1 as a stem cell marker. 


\section{DCLK1: a gastrointestinal brush cell marker?}

Brush cells, also known as tuft cells, express both neurogenic and inflammatory markers [17]. These cells are found in both the human and murine normal and neoplastic intestinal mucosa, as well as in mucosal inflammation [18,19]. In 2008, a group investigating the function of the Trpm5-expressing brush cells of the gastrointestinal tract, found high levels of DCLK1 transcript in these cells [17]. Based on these findings and on the observation that the majority of DCLK1 positive cells were found in mouse villi and not in the stem cell zone, Gerbe and colleagues contested the use of DCLK1 as a gastrointestinal stem cell marker cells, and showed that DCLK1 cells expressed COX 1 and 2 enzymes and a large amount of villin and tubulin, which are typical of brush cells [20]. These authors further identified a unique signature marker of brush cells, which included SOX9, COX1, COX2, hematopoietic prostaglandin-D synthase, and DCLK1 [21]. Moreover, $98 \%$ of DCLK1 cells coexpressed COX1 and can be recognized as brush cells by their high expression of $\mathrm{SOX}$, a brush cell signature marker common to enteroendocrine cells. Interestingly, SOX9 is also a putative gastrointestinal stem cell marker [22-24].

Further evidence challenging DCLK1 as a gastrointestinal stem cell marker cells stemmed from a label retention assay that demonstrated that DCLK1 positive cells do not retain label after 2 weeks. This conflicted with early reports using a shorter modified assay with radiation [10], and therefore an attitude of caution seemed to develop regarding whether DCLK1 cells were legitimate quiescent stem cells [25]. Furthermore lineage tracing studies of Lgr 5 expressing cells showed staining for DCLK1 and SOX 9 among the Lgr5 positive clones, thereby demonstrating that DCLK1 positive cells originate from Lgr5-expressing transient amplifying cells. With a tamoxifen inducible Atoh1 knock out mouse Gerbe and colleagues demonstrate that DCLK1 cells are Atoh1 dependant and therefore belong to the granulocitic cell lineage, a finding that was not confirmed by others [26]. Finally, in the tamoxifen inducible neurogenin3, Gfi-1, Spdef and Sox9 knockout mice, there is persistence of DCLK1 cells, demonstrating that their differentiation requirements are different from the ones of enteroendocrine, paneth and goblet cells [21]. The notion that DCLK1 was not a stem cell marker but a brush cell created great interest in the research community.

\section{DCKL1: expressed in enteroendocrine cells}

Brush cells are known to express many signaling peptides common to enteroendocrine cells suggesting that they are a type of enteroendocrine cell [27]. In order to distinguish brush cells from enteroendocrine cells Bjerknes and colleagues performed a series of lineage tracing and co-localization studies that included the marker DCKL1. First of all, these investigators demonstrated that brush cells first express the brush cell progenitor marker Gfi1b while DCLK1 is expressed at a later stage of differentiation [26]. DCLK1 is also expressed in a subset of cells expressing the enteroendocrine lineage marker
Insm1. Based on this data, 2 types of DCLK1 positive cells were identified; one co-expressing enteroendocrine markers and the other co-expressing brush cells markers including Gfi1 b and UEA-1. These 2 types of cells can also be distinguished by their morphologic appearance on confocal microscopy since enteroendocrine DCLK1 positive cells are flask shaped while brush cells have a volumetrically thin neck. In addition to the brush cell and the enteroendocrine cell type, there was small subset of immature mitotic DCLK1 positive cells at the crypt base. Finally the same investigators showed that brush cells are Atoh 1 independent by demonstrating an increase of DCLK1 positive cells after conditional Atoh1 deletion in transgenic mouse. It is clear from these findings that DCLK1 expression is not limited to brush cells. Nevertheless, DCLK1 has been increasingly used to investigate brush cells and their characteristics $[18,25,28-30]$.

If DCLK1 was exclusively a marker of differentiated brush cells, why do DCLK1 positive cells also exhibit enteroendocrine and stem cell features? More insight into the enteroendocrine and stem cell characteristics of DCLK1 positive cells were gained through a series of experiments investigating the fate of enteroendocrine progenitors in cholecystokinin-green fluorescent protein transgenic mice. In this study, Sei and colleagues described a subset of enteroendocrine cells located at the base of small intestinal crypts that were positive for the stem cell markers CD133 and LGR5 as well as DCLK1 [31]. These crypt base cells showed intermediate features between stem cells and mature enteroendocrine cells, since they express $\mathrm{CgA}$, which is a marker of well-differentiated endocrine cells, as well as Neurogenin 3 and multiple hormones typical of enteroendocrine progenitors. This and other experiments led our laboratory to investigate DCLK1 expression in the normal human colon. In this study, we showed that DCLK1 positive cells were present in $60 \%$ of colonic crypts and were mostly located in crypt positions 1-4 (unpublished data). Morphologically these cells resemble enteroendocrine cells and had a strong cytoplasmic immunostaining that was antiluminal in distribution (Figure 2). Interestingly, a subpopulation of DCLK1 cells located at the crypt base co-stained for LGR5. We also demonstrated CgA immunoreactivity in all DCKL1 positive cells throughout the crypt, while CgA positive/DCLK1 negative cells were found only in the upper part of the crypt (unpublished data). Our results suggest that in normal human colon all DCLK1 cells co-express enteroendocrine markers and that a subset of these cells located at the crypt base are LGR5 positive progenitor cells.

\section{DCLK1: present in both differentiated cells and stem cells of the small intestine}

The explanation of why DCLK1 positive brush cells also appeared to have stem cell characteristics was, in part, provided by the Clevers group who used a three-color single-molecule fluorescence in situ hybridization technique to study a comprehensive panel of intestinal stem-cell markers during 


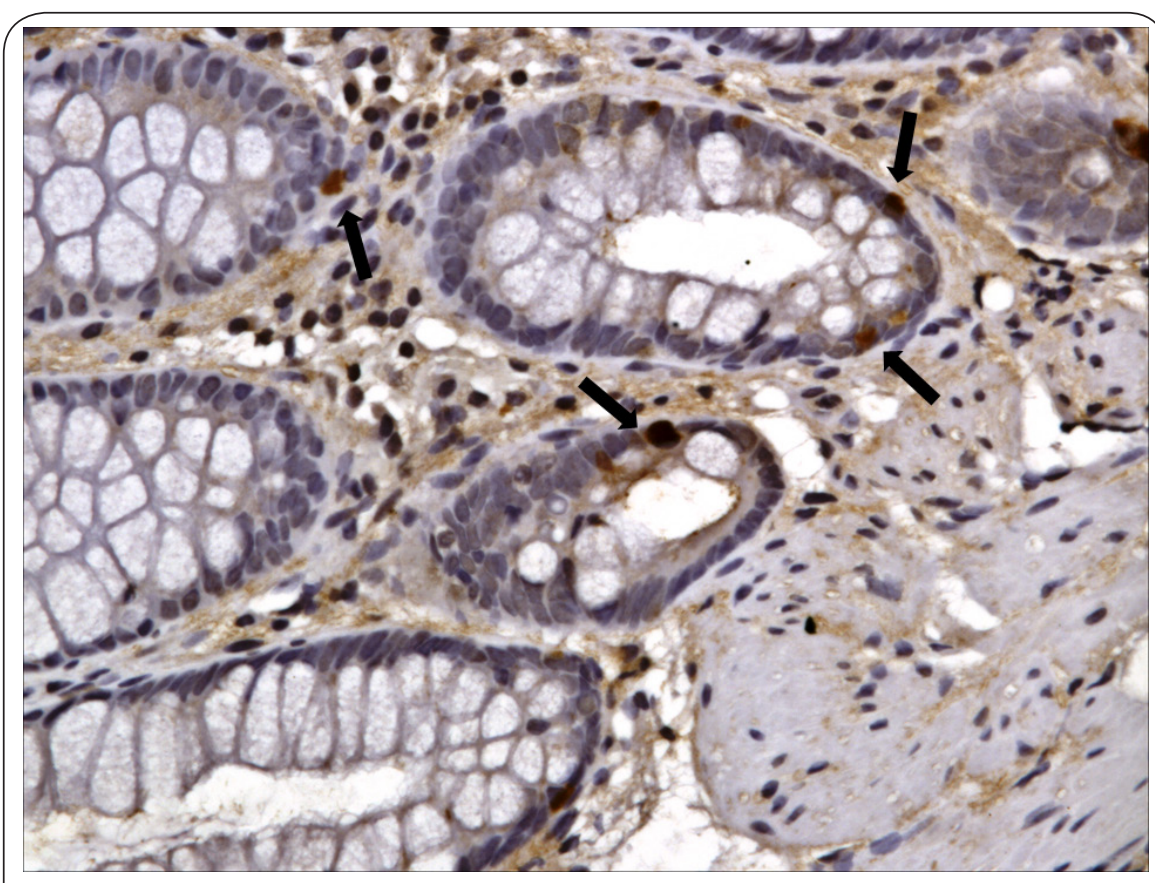

Figure 2. DCLK1 immunoreactivity in the normal human colon. DCLK1 positive cells are found at the base of a normal colonic crypt. Immuno-staining is anti-luminal (arrows) and cells morphologically resemble enteroendocrine cells . (200x).

homeostasis and regeneration [28]. By using this approach, gene co-expression in single cells were measured with a correlation coefficient. A high correlation coefficient would identify genes that could be regulated by a common upstream gene or directly regulate each other while a low correlation coefficient would represent mutually exclusive genes. A striking finding from this study was that Dclk1 expression, at all crypt positions, significantly correlated with that of all the stem-cell markers that were otherwise confined to the crypt bottom, including Lgr5, Sox9, Musashi-1, EphB2, and EphB3. To assess the capacity of Dclk1 expressing cells to regenerate the whole crypt their response after $12 \mathrm{~Gy}$ and $1 \mathrm{~Gy}$ of irradiation was measured. Based on the low Ki67 expression, their turnover similar to goblet cells and their co-expression of COX1, a brush cell marker, DCLK1 positive cells did not seem to contribute to crypt cell regeneration after a sterilizing dose of radiation and after $1 \mathrm{~Gy}$ did not show the expected apoptotic response [6]. A better explanation of DCLK1 stem cell function was demonstrated in a recent FACS isolation and characterization study of small intestinal cells expressing the putative intestinal stem cell marker Sox9. These studies were prompted by the observations made in the Sox9-EGFP-reporter mouse model that intestinal stem cells expressed low levels of Sox9-EGFP while enteroendocrine cells expressed high levels of Sox9-EGFP [32]. Ramalingam and colleagues recently confirmed these findings in a series of experiments using the same mouse model. These investigators showed that cells expressing low levels of Sox9 (Sox9-low cells) were enriched with Lgr5 mRNA and that cells expressing a high level of Sox9 (Sox9-high cells) were enriched with enteroendocrine hormones. However, Sox9-high cells were also the only intestinal cells expressing the markers of quiescent stem cells such as Bmi1 and Hopx as well as Dclk1, whose transcript was 147 fold higher in Sox9-high compared to Sox9-low cells. Surprisingly, while Sox9-low stem cells showed the predicted expansion and hyper-proliferation after radiation, Sox9-high cells were also found to be proliferating and expanding, albeit at a slower pace. Interestingly, while Sox9 low cells were able to form multi-lineage organoids in vitro both before and after irradiation, Sox9 high cells were able to do so only after irradiation, thus, leading to the hypothesis that Sox9-high cells are quiescent intestinal stem cells activated in stress condition. In support of this hypothesis, the analysis of Sox9-high cells gene expression before and after irradiation showed a up-regulation of genes required for quiescent cells to re-enter the cell cycle, genes associated with DNA repair, and other genes associated with resistance to apoptosis and with proliferation, which are typically found in activated stem cells. Many of the up-regulated genes were also linked to radioresistance and cancer growth. This dual enteroendocrine and quiescent stem cell nature of Sox9-high cells, together with their increased Dclk1 expression may well explain some of the previous contradictory results, such as the presence of stem cell antigens in well differentiated brush or enteroendocrine cells or the different involvement, compared to Lgr5-expressing stem cells, in the regeneration process after irradiation. The spatial relationship of DLCK1 positive cells with other cells in the intestinal crypt is shown in Figure 3. 


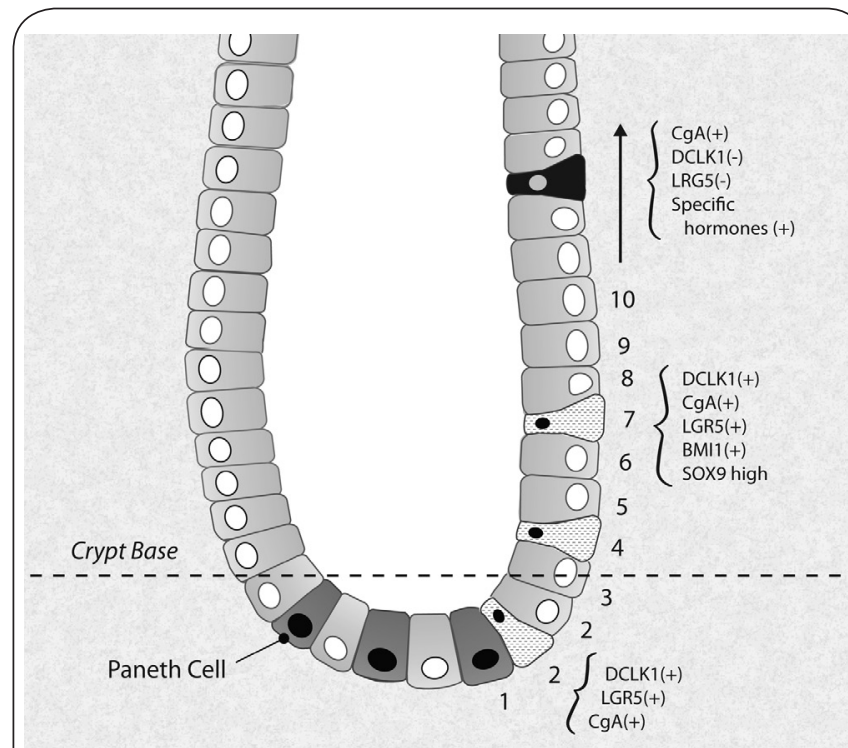

Figure 3. Localization of DCLK1 positive cells along the intestinal crypt. DCLK1 positive cells co-express neuroendocrine markers and can be found either at the crypt base among the Paneth cells or above the crypt base in an area which includes the +4 compartment were quiescent stem cells are thought to reside. Crypt base DCLK1 positive cells are also positive for LGR5 while DCLK1 positive cells above the crypt base are LGR 5 negative but may express other markers of quiescent stem cells such as SOX9 and BMI1. In the upper part of the crypt a mature neuroendocrine cell is depicted.

\section{DCLK1 expression in the stomach}

Although DCLK1 expression is well characterized in the small intestine, its expression in the gastric mucosa is not well defined. In the stomach, DCLK1 cells are found in the stem cell zone located at the isthmus of the fundic glands [33] and are clearly distinct from TFF2 transcript-expressing cells, which are considered the progenitor cells for mucus neck, parietal and zymogenic cells $[12,34]$. Gastric DCLK1 cells express parietal cell markers and partially co-express MSI1. In rodent models of acute gastritis and of chronic ulcer DCLK1 cells expand beyond the stem cell zone but remain PCNA negative $[33,34]$. In these conditions as well as after irradiation, DCLK1 cells are found adjacent to the PCNA positive compartment, suggesting a possible role in the restitution of mucosal integrity $[33,34]$.

\section{DCLK1 expression in the pancreas}

In adult mice DCLK1 is expressed in the ductal epithelial cells and islets of the pancreas, but not within acinar cells [35]. Coexpression was observed with Neurogenin 3, a pancreatic stem cell marker highly expressed during embryogenesis, and with somatostatin, indicating an endocrine phenotype, but not with glucagon or insulin. FACS-isolated DCLK1 positive cells formed spheres in low attachment conditions and, when injected into nude mice, gave rise to nodular growths expressing the markers of early pancreatic development (PDX-1), glandular epithelium (cytokeratin-14 and Ep-CAM), and islet-like structures (somatostatin and secretin) [35]. This suggests that pancreatic DCLK1 positive cells are undifferentiated stem-like cells that can give rise to all pancreatic lineages.

\section{Regulation of DCLK1 in the gastrointestinal tract}

The mechanisms regulating the homeostasis of DCLK1 intestinal cells are incompletely understood. The balance between notch signal, leading to enterocyte differentiation, and ATOH signal leading to the granulocytic lineage regulates the fate of intestinal stem cells. To date, studies on Atoh dependence of DCLK1 cells have been conducted in different mouse models leading to opposite results as outlined above $[21,26]$. However using the GFP-Lgr5 mouse, it has recently been shown that Notch inhibition (and therefore Atoh 1 increase) results in a decrease in Lgr5 expressing-cells and in an increase in the differentiation and number of secretory lineage cells including DCLK1 positive cells [30]. It has been hypothesized that DCLK1 cells are only partially dependant on Atoh1, which would explain these conflicting results [26]. Moreover, directly blocking the cell division through $\mathrm{Cdc} 25$ loss induces the differentiation of crypt base stem cells but not that of DCLK1 and Musashi-1 positive cells located above the base, which suggests that these cells are independent from cell cycle regulation [11].

Wnt and KIf4 are two key regulators of stem cell self-renewal that are necessary in the maintenance of the undifferentiated state. By using the Wnt reporter mouse model, investigators have shown that Wnt responsive cells are DCLK1 negative [16]. Moreover, in transgenic mice with a tamoxifen-induced partial depletion of the Klf4 transcription factor, a decrease of goblet cells and an increase of DCLK1 positive cells with expansion beyond the crypt stem cell zone and loss of cell polarity has been demonstrated [14]. Interestingly, while Klf4 is known to play an important role in cell fate reprogramming and self-renewal, it is also known as a tumor suppressor gene, which is down regulated in colorectal cancer.

\section{DCLK1 in gastrointestinal neoplasia}

The propagation and growth capacity of many human cancers is thought to be driven, in part, by a subset of undifferentiated cells with stem-like features called cancer stem cells (CSC) or cancer initiating cells [36]. One notion is that these CSC originate from tissue resident stem cell populations that have undergone malignant transformation [6]. In fact, some of the markers of intestinal stem cells such as LGR5, MSI1 and DCLK1, found in the normal intestinal crypts, are over-expressed in tumors $[37,38]$. However, to date the relationship between CSC and the tissue resident stem cell populations' remains poorly understood. 


\section{DCLK1 in gastrointestinal carcinogenesis: animal data Colon}

In Apc-mutant mice, DCLK1 positive cells were shown to expand beyond the stem cell compartment to the villi [9]. Similar observations of expansion beyond the stem cell compartment were also reported in the colon of progastrin overexpressing transgenic mice [39]. These mice develop colonic hyperproliferation and colorectal cancer through a decrease in apoptosis and an increase in $\beta$-catenin and crypt fission. Deletion or inhibition of the gastrin receptor CCK2R decreased the tumor formation as well as the number of DCLK1 positive cells. Similar findings were observed in the mouse colon after progastrin administration as well as in an in vitro model of progastrin autocrine stimulation that relies on the presence of the annexin 2 receptor on cancer cells [40]. Another interesting observation in Apc-mutant mice was the fact that abundant DCKL1 positive cells were noted in the areas surrounding the adenomas but were rare within adenoma itself [9]. However, within the adenomas DCLK1 positive cells were found to have nuclear $\beta$-catenin translocation, which is a sign of neoplastic activation $[9,21]$. Similar findings were reported in adenomas from mice carrying a K-ras mutation [21].

Studies involving radiation injury provide an illustration of the complex function of DCKL1 in the colon. For instance, in the progastrin over-expressing mouse model, DCLK1 positive cells were resistant to radiation [39]. Interestingly, irradiated mice receiving recombinant regenerating islet-derived protein 4 (REG4), a protein expressed in colorectal cancer, are resistant to radiation-induced apoptosis and show a marked increase of DCLK1 positive cells, suggesting a possible role of these cells in radioresistance. Furthermore, strategies aimed at antagonizing Reg 4 resulted in a decrease in tumor growth and an increase in the tumors response to radiotherapy [41].

\section{Esophagus}

In a transgenic mouse model, IL-1 $\beta$ over-expression in the mouse esophagus has been shown to induce IL- 6 dependent Barrett esophagus and esophageal adenocarcinoma [42]. In these mice, abundant DCLK1 positive cells were present in the gastric cardia adjacent to the area of metaplasia. However, with progression to dysplasia, Dclk1 expression was downregulated. In a H+/K+-ATPase-IL-1 $\beta$ transgenic mouse model, the expansion of cells expressing DCLK1 was strongly associated with inflammation-related carcinogenesis and preceded the development of gastric cancer. Notably, over-expression or infusion of IFN- $\gamma$ reduces both cell proliferation and the number of DCLK1 positive cells and therefore raises the possibility of a direct effect of IFN- $\gamma$ on gastric progenitor cells [43].

\section{Pancreas}

DCLK1 cells are significantly increased in the pancreatic tissue of K-ras transgenic mice that develop very aggressive metastatic pancreatic cancers. Of note, this increase in DCKL1 cells directly correlated with the neoplastic changes [44]. Moreover in this tumor model, DCLK1 cells co-localized with the pancreatic cancer cell marker 14-3-3 $\sigma$ protein in both preneoplastic and neoplastic lesions. Based on these reports the sobering conclusion is that DCLK1 has an active role in gastrointestinal carcinogenesis.

\section{DCLK1 in gastrointestinal carcinogenesis: human data Cell lines}

DCLK1 has been shown to be expressed in human colon [45,46], pancreatic [44] and hepatocellular carcinoma [47] cell lines as well as in tumor xenografts established from these cell lines. Recently, using flow cytometry, only a small fraction $(0.5-2 \%)$ of the Huh7 hepatocellular carcinoma cell line had a high surface expression of DCLK1. However, if these DCLK1 positive cells were isolated by fluorescence-activated cell sorting and plated they formed spheroids while the DCLK1 negative pool did not, implying that surface expression of DCLK1 is related to CSC properties [47]. Moreover, in the Huh7 cells, DCLK1 transcripts increase after HCV transfection and after induction of pluripotency, suggesting that DCLK1 may play a role in $\mathrm{HCV}$ related carcinogenesis and may be a new hepatocellular carcinoma CSC marker [47].

\section{Colon}

Data on DCLK1 expression in human tumors is sparse. Using immunohistochemistry, studies of tissue microarrays from colon, breast, pancreatic and prostate cancers showed an increased expression of DCLK1 in the epithelial cells and in the cancer associated stroma compared to paired normal tissue [45,46]. Our laboratory has recently investigated DCLK1 immunoreactivity [48] in the colorectal adenoma- carcinoma sequence from 71 patients. DCLK1 immunoreactivity was found in more that $70 \%$ of the colorectal adenomas and carcinomas and in more that $50 \%$ of the distant metastasis. Immunohistochemical staining of DCLK1 in a colorectal carcinoma is shown in Figure 4. In adenomas, strong immunoreactivity was only present in those with high-grade dysplasia. In colorectal cancers, strong immunoreactivity was significantly associated with recurrent disease. Interestingly, there was no correlation between DCLK1 immunoreactivity and tumor stage, indicating that DCLK1 may be an independent prognostic factor. Taken together these findings indicate that DCLK1 may be a marker of advanced adenomas and a useful prognostic marker to identify colorectal carcinomas at high risk for recurrence. However, larger studies are needed to confirm these findings.

\section{Esophagus}

DCLK1 expression in biopsy samples taken from Barrett's mucosa and esophageal adenocarcinoma has been investigated in two human studies [42,49]. These studies showed a significant increase in the DCLK1 immunoreactivity and the mRNA levels in Barrett's esophagus (BE) compared to normal squamous 


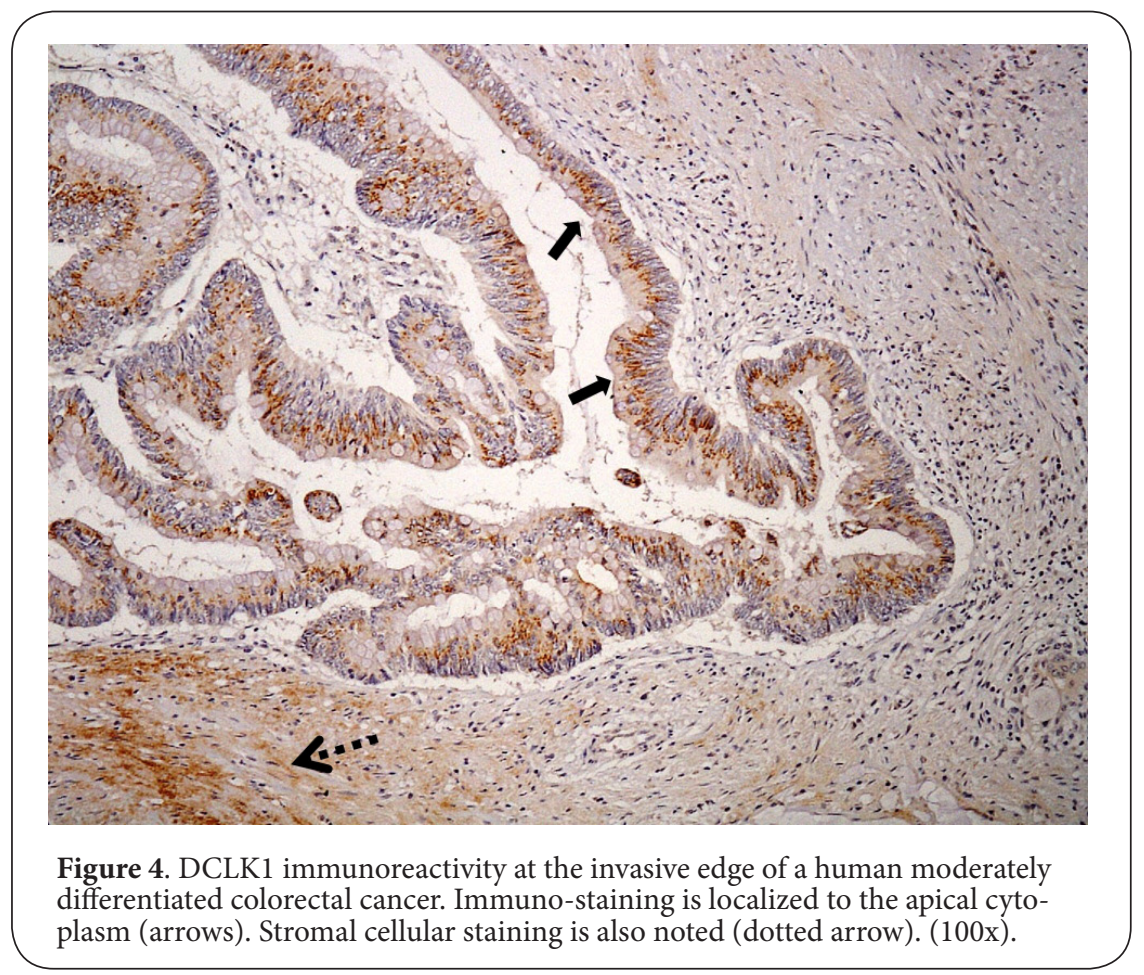

epithelium. In esophageal adenocarcinoma, an increased stromal DCLK1 staining was noted compared to adjacent epithelia and in one study a progressive increase of DCLK1 expression in $\mathrm{BE}$ from dysplasia to adenocarcinoma was observed [49]. Interestingly, LGR5 and DCLK1 were significantly elevated in the gastric cardia of BE patients [42]. The upregulation of gastric columnar progenitor cells in the region of the cardia and in $\mathrm{BE}$ suggest that the metaplastic lineage in $\mathrm{BE}$ lesions in humans may be derived from a gastric cardia lineage. However, based on the current level of evidence, it is not clear if DCLK1 can be clinically useful to predict progression from $\mathrm{BE}$ to esophageal adenocarcinoma.

\section{Pancreas}

Although pancreatic tumors show strong DCLK1 immunoreactivity in the ductal cells and stroma [44], DCLK1 is also present within the islets of the normal pancreas, and in the pancreatic ducts and stroma of patients with chronic pancreatitis. Among adenocarcinomas, co-localization with the pancreatic intraductal neoplasia marker 14-3-3 s was observed, suggesting that neoplastic transformation may arise in putative pancreatic CSCs. Sureban and colleagues, also characterized the DCLK1 positive stromal cells using the mesenchymal lineage marker vimentin, and showed that DCLK1 immunoreactivity was present in $10 \%$ of vimentin positive stromal cells, suggesting that DCAMKL-1 may be involved in the desmoplastic reaction associated with human pancreatic cancer and may also play a role in epithelial-mesenchymal transition (EMT) [44].

\section{Liver}

DCLK1 immunoreactivity has recently been evaluated in liver tissue microarrays of hepatocellular carcinoma and advanced cirrhosis with and without carcinoma, as well as in liver biopsies from patients with HCV positive cirrhosis [47]. The results obtained from the analysis of the tissue microarrays demonstrated an increased DCLK1 expression in hepatocytes, bile ducts and stromal tissue in parallel with the neoplastic progression from normal liver to hepatocellular carcinoma. By comparison, the liver biopsies from patients with HCV positive cirrhosis showed a sporadic expression of DCLK1. However, marked DCLK1 immunoreactivity and CK19 staining (hepatic tumor marker) were observed in one patient with uncontrolled $\mathrm{HCV}$ proliferation and dysplastic regenerative nodules.

\section{DCLK1: a potential target of new therapeutic strategies?}

A single research group has explored the possibility of using DCLK1 as a tumor target in different gastrointestinal cancer models. These investigators showed that the intra-tumoral injection of DCLK1 siRNA resulted in a 55\% reduction in the size of colorectal cancer xenografts [45]. The effect of DCLK1 on tumor growth appeared to be related to its inhibition of Let7A miRNA as well as a concomitant increase in the Let7A target c-myc [45]. Interestingly, an inverse relation between DCLK1 and Let7A miRNA was also observed in normal mouse intestinal cells, suggesting that this existing pathway may become activated in tumors [45]. Moreover, using a xenograft tumor model Sureban and colleagues demonstrated a decrease of Notch 1 mRNA in the xenografts treated with nanoparticle-encapsulated DCLK1 

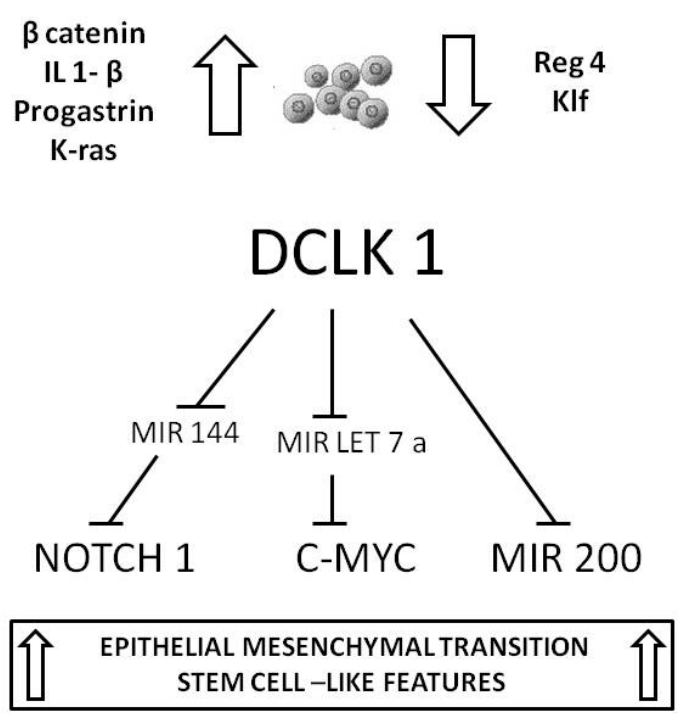

Figure 5. Factors regulating the proliferation of DCLK1 positive cells in gastrointestinal neoplasia, and downstream targets mediating the effects of DCLK1 on tumor growth.

siRNA and an increase of Notch1 inhibitor miR-144, suggesting that, in colorectal tumors, DCLK1 may activate Notch1 via a miR144 dependant mechanism [46]. Also, in this study the potent epithelial-mesenchymal transition (EMT) inhibitor miR-200 was decreased in the siRNA treated tumors, an effect which was not seen with NOTCH1 inhibition alone. EMT is a phenotypic conversion necessary for embryonic development and wound healing, which is also found in neoplasia where it is associated with disease progression and outcome. Tumors after DCLK1 siRNA treatment also showed the down-regulation of ZEB1, ZEB2, Snail and Slug and an up-regulation of E-cadherin, which are consistent with the hypothesis that in human colorectal cancer knockdown of DCLK1 may inhibit EMT via a miR-200adependent mechanism. Similar results were also shown after siRNA-mediated knockdown in a human pancreatic cancer cell line [44] suggesting that DCLK1 may be a valid tumor target in for the treatment of other aggressive tumors of the gastrointestinal tract. Recently using a hepatocellular carcinoma cell line transfected with HCV, Ali and colleagues showed that siRNA-mediated inhibition of Dclk1 resulted in the decrease of HCV RNA and the viral polymerase levels, suggesting a lower viral replication rate and a possible therapeutic role of inhibiting Dclk1 expression as a potential treatment of HCV induced carcinogenesis [47]. The current known information regarding how DCLK1 is regulated and how DCLK1 regulates other molecules / pathways to drive carcinogenesis is illustrated in Figure 5.

\section{Future directions and conclusion}

Since the first description of DCLK1 in the gastrointestinal tract, a lot of progress has been made in our understanding of this gene expression. However numerous questions remain unanswered regarding the origin and fate of gastrointestinal DCLK1 positive cells. The presence of DCLK1 in brush cells does not explain its association with other stem cell marker or its activation after radiation injury. Lineage tracing experiments may shed more light on whether a subset of DCLK1 cells may have stem cell properties different from those of Lgr5-positive transient amplifying cells and whether they may be indeed quiescent stem cells. Moreover, brush cells have not been shown to be increased in human gastrointestinal cancer [21] whereas there is accumulating evidence that DCLK1 is not only over expressed in several human malignancies but it also may play a functional role in gastrointestinal carcinogenesis. At present it remains unknown whether DCLK1 can be used a CSC marker or as a therapeutic target largely because the role and function of DCLK1 in tumor relapse and metastasis remain incompletely explored. CSCs are involved in resistance to conventional chemotherapy and radiotherapy and have been related to the ability of cancers to metastasize [36]. The increased expression of DCLK1 has been shown in a small number of studies to be directly related to both radioresistance [23,41] and tumor aggressiveness [48]. These studies can be interpreted as evidence to support the development of therapeutic strategies beyond traditional chemotherapeutic gents. 


\section{List of abbreviations:}

Apc: adenomatous polyposis coli

Atoh1: atonal homolog 1

BE: Barrett's esophagus

BRDU: broxo-deoxy uridine

CgA: chromogranin A

CK19: cytokeratin 19

COX 1 and 2: cyclo-oxygenase 1 and 2

CSC: cancer stem cell

DCLK1: doublecortin-like kinase 1

EMT: epithelial-mesenchymal transition

Gfi-1b: growth factor independent 1B transcription repressor

Gy: Gray

HCV: hepatitis $C$ virus

IFN- $\gamma$ : interferon $\gamma$

IL-6: interleukin 6

IL-1 $\beta$ : interleukin $1 \beta$

LGR5: Leucine-rich repeat-containing G-protein coupled receptor 5 miRNA: micro RNA

MSI1: musashi 1

NOTCH1: Notch homolog 1

PCNA: proliferating cell nuclear antigen

siRNA: small interfering RNA

Sox9: SRY (sex determining region Y)-box 9

Spdef: SAM (sterile alpha motif) pointed domain

containing ETS (E-twenty six) transcription factor

Trpm5: Transient receptor potential cation

channel subfamily $\mathrm{M}$ member 5

UEA-1: ulex Europeus 1

ZEB 1 and 2: Zinc finger E-box-binding homeobox 1 and 2

\section{Competing interests}

Dr Bellows receives grant support from the Life Cell

corporation and is also on their speakers bureau.

\section{Authors' contributions}

The work presented here was carried out in collaboration between both authors. CB and GG defined the research theme as well as interpreted the results and wrote the paper. All authors have contributed to, seen and approved the manuscript.

\section{Publication history}

Editors: Fei Chen, Wayne State University, USA.

Stephanie Ma, The University of Hong Kong, Hong Kong.

EIC: G.J. Peters, VU University Medical Center, Netherlands.

Received: 24-Apr-2012 Revised: 07-May-2012

Accepted: 04-June-2012 Published: 11-June-2012

\section{References}

1. Dijkmans TF, van Hooijdonk LW, Fitzsimons CP, Vreugdenhil E: The doublecortin gene family and disorders of neuronal structure. Cent Nerv Syst Agents Med Chem 2010; 10;(1.);32-46. I Article I PubMed

2. Burgess $\mathrm{HA}$, Reiner $\mathrm{O}$ : Alternative splice variants of doublecortin-like kinase are differentially expressed and have different kinase activities. $J$ Biol Chem 2002; 277;(20.);17696-705. | Article | PubMed

3. Sossey-Alaoui K, Srivastava AK: DCAMKL1, a brain-specific transmembrane protein on 13q12.3 that is similar to doublecortin (DCX). Genomics 1999; 56;(1.);121-6. | Article | PubMed

4. Omori Y, Suzuki M, Ozaki K, Harada Y, Nakamura Y, Takahashi E, et al.: Expression and chromosomal localization of KIAA0369, a putative kinase structurally related to Doublecortin. J Hum Genet 1998;

\section{3;(3.);169-77. | Article | PubMed}

5. Giannakis M, Stappenbeck TS, Mills JC, Leip DG, Lovett M, Clifton SW, et al.: Molecular properties of adult mouse gastric and intestinal epithelial progenitors in their niches. J Biol Chem 2006; 281;(16.);11292-300. | Article | PubMed Abstract | PubMed Full Text

6. Barker N, Ridgway RA, van Es JH, van de Wetering $M$, Begthel $H$, van den Born $\mathrm{M}$, et al.: Crypt stem cells as the cells-of-origin of intestinal cancer Nature 2009; 457;(7229.);608-11. | Article | PubMed

7. Montgomery RK, Carlone DL, Richmond CA, Farilla L, Kranendonk ME, Henderson DE, et al.: Mouse telomerase reverse transcriptase (mTert) expression marks slowly cycling intestinal stem cells. Proc Natl Acad Sci U S A 2011; 108;(1.);179-84. | Article I PubMed Abstract | PubMed Full Text

8. Barker N, Bartfeld S, Clevers H: Tissue-resident adult stem cell populations of rapidly self-renewing organs. Cell Stem Cell 2010; 7;(6.);656-70. I Article | PubMed

9. May R, Riehl TE, Hunt C, Sureban SM, Anant S, Houchen CW: Identification of a novel putative gastrointestinal stem cell and adenoma stem cell marker, doublecortin and CaM kinase-like-1, following radiation injury and in adenomatous polyposis coli/multiple intestinal neoplasia mice. Stem Cells 2008; 26;(3.);630-7. I Article I PubMed

10. May R, Sureban SM, Hoang N, Riehl TE, Lightfoot SA, Ramanujam R, et al.: Doublecortin and CaM kinase-like-1 and leucine-rich-repeat-containing G-protein-coupled receptor mark quiescent and cycling intestinal stem cells, respectively. Stem Cells 2009; 27;(10.);2571-9. | Article | PubMed Abstract | PubMed Full Text

11. Lee G, White LS, Hurov KE, Stappenbeck TS, Piwnica-Worms H: Response of small intestinal epithelial cells to acute disruption of cell division through CDC25 deletion. Proc Natl Acad Sci U S A 2009; 106;(12.);4701-6. | Article | PubMed Abstract | PubMed Full Text

12. Quante M, Marrache F, Goldenring JR, Wang TC: TFF2 mRNA transcript expression marks a gland progenitor cell of the gastric oxyntic mucosa. Gastroenterology 2010; 139;(6.);2018-27 e2. | Article | PubMed Abstract I PubMed Full Text

13. Samuel S, Walsh R, Webb J, Robins A, Potten C, Mahida YR: Characterization of putative stem cells in isolated human colonic crypt epithelial cells and their interactions with myofibroblasts. Am J Physiol Cell Physiol 2009; 296;(2.);C296-305. | Article | PubMed Abstract | PubMed Full Text

14. Yu T, Chen X, Zhang W, Li J, Xu R, Wang TC, et al.: Kruppel-like factor 4 regulates intestinal epithelial cell morphology and polarity. PLOS One 2012; 7;(2.);e32492. | Article | PubMed Abstract | PubMed Full Text

15. Dekaney CM, Gulati AS, Garrison AP, Helmrath MA, Henning SJ: Regeneration of intestinal stem/progenitor cells following doxorubicin treatment of mice. Am J Physiol Gastrointest Liver Physiol 2009; 297;(3.);G461-70. | Article | PubMed Abstract | PubMed Full Text

16. Davies PS, Dismuke AD, Powell AE, Carroll KH, Wong MH: Wnt-reporter expression pattern in the mouse intestine during homeostasis. $B M C$ Gastroenterol 2008; 8;(57). | Article | PubMed Abstract | PubMed Full Text

17. Bezencon C, Furholz A, Raymond F, Mansourian R, Metairon S, Le Coutre $\mathrm{J}$, et al.: Murine intestinal cells expressing Trpm 5 are mostly brush cells and express markers of neuronal and inflammatory cells. J Comp Neurol 2008; 509;(5.);514-25. | Article | PubMed Abstract

18. Saqui-Salces M, Keeley TM, Grosse AS, Qiao XT, El-Zaatari M, Gumucio DL, et al.: Gastric tuft cells express DCLK1 and are expanded in hyperplasia. Histochem Cell Biol 2011; 136;(2.);191-204. I Article I PubMed

19. Morroni M, Cangiotti AM, Cinti S: Brush cells in the human duodenojejunal junction: an ultrastructural study. J Anat 2007; 211;(1.);125-31. | Article | PubMed Abstract | PubMed Full Text

20. Gerbe F, Brulin B, Makrini L, Legraverend C, Jay P: DCAMKL-1 expression identifies Tuft cells rather than stem cells in the adult mouse intestinal epithelium. Gastroenterology 2009; 137;(6.);2179-80; author reply 80-1. | Article | PubMed

21. Gerbe F, van Es JH, Makrini L, Brulin B, Mellitzer G, Robine S, et al.: Distinct ATOH1 and Neurog3 requirements define tuft cells as a new secretory cell type in the intestinal epithelium. J Cell Biol 2011; 
192;(5.);767-80. | Article | PubMed Abstract | PubMed Full Text

22. Formeister EJ, Sionas AL, Lorance DK, Barkley CL, Lee GH, Magness ST: Distinct SOX9 levels differentially mark stem/progenitor populations and enteroendocrine cells of the small intestine epithelium. Am J Physiol Gastrointest Liver Physiol 2009; 296;(5.);G1108-18. | Article | PubMed Abstract | PubMed Full Text

23. Ramalingam S, Daughtridge GW, Johnston MJ, Gracz AD, Magness ST: Distinct levels of Sox9 expression mark colon epithelial stem cells that form colonoids in culture. Am J Physiol Gastrointest Liver Physiol 2012; 302;(1.);G10-20. | Article | PubMed

24. Van Landeghem L, Santoro MA, Krebs AE, Mah AT, Dehmer JJ, Gracz AD, et al.: Activation of two distinct Sox9-EGFP-expressing intestinal stem cell populations during crypt regeneration after irradiation. Am J Physiol Gastrointest Liver Physiol 2012; 302;(10.);G1111-32. | Article I PubMed

25. Escobar M, Nicolas $P$, Sangar F, Laurent-Chabalier $S$, Clair $P$, Joubert $D$, et al.: Intestinal epithelial stem cells do not protect their genome by asymmetric chromosome segregation. Nat Commun 2011; 2;(258. | Article | PubMed Abstract I PubMed Full Text

26. Bjerknes M, Khandanpour C, Moroy T, Fujiyama T, Hoshino M, Klisch $\mathrm{TJ}$, et al.: Origin of the brush cell lineage in the mouse intestinal epithelium. Dev Biol 2012; 362;(2.);194-218. | Article | PubMed

27. Bjerknes M, Khandanpour C, Moroy T, Fujiyama T, Hoshino M, Klisch $\mathrm{TJ}$, et al.: Origin of the brush cell lineage in the mouse intestinal epithelium. Dev Biol 2012; 362;(2.);194-218. | Article | PubMed

28. Itzkovitz S, Lyubimova A, Blat IC, Maynard M, van Es J, Lees J, et al.: Single-molecule transcript counting of stem-cell markers in the mouse intestine. Nat Cell Biol 2012; 14;(1.);106-14. I Article I PubMed

29. Nam KT, O'Neal R, Lee YS, Lee YC, Coffey RJ, Goldenring JR: Gastric tumor development in Smad3-deficient mice initiates from forestomach/ glandular transition zone along the lesser curvature. Lab Invest 2012. | Article I PubMed

30. VanDussen KL, Carulli AJ, Keeley TM, Patel SR, Puthoff BJ, Magness ST, et al.: Notch signaling modulates proliferation and differentiation of intestinal crypt base columnar stem cells. Development 2012; 139;(3.);488-97. | Article | PubMed

31. Sei Y, Lu X, Liou A, Zhao X, Wank SA: A stem cell marker-expressing subset of enteroendocrine cells resides at the crypt base in the small intestine. Am J Physiol Gastrointest Liver Physiol 2011; 300;(2.);G345-56. | Article | PubMed Abstract | PubMed Full Text

32. Gracz AD, Ramalingam S, Magness ST: Sox9 expression marks a subset of CD24-expressing small intestine epithelial stem cells that form organoids in vitro. Am J Physiol Gastrointest Liver Physiol 2010; 298;(5.);G590-600. | Article | PubMed Abstract | PubMed Full Text

33. Zhang $Y$, Huang $X$ : Investigation of doublecortin and calcium/ calmodulin-dependent protein kinase-like-1-expressing cells in the mouse stomach. J Gastroenterol Hepatol 2010; 25;(3.);576-82. | Article I PubMed

34. Kikuchi M, Nagata H, Watanabe N, Watanabe H, Tatemichi M, Hibi T: Altered expression of a putative progenitor cell marker DCAMKL1 in the rat gastric mucosa in regeneration, metaplasia and dysplasia. $B M C$ Gastroenterol 2010; 10;65. | Article | PubMed Abstract | PubMed Full $\underline{\text { Text }}$

35. May R, Sureban SM, Lightfoot SA, Hoskins AB, Brackett DJ, Postier RG, et al.: Identification of a novel putative pancreatic stem/progenitor cell marker DCAMKL-1 in normal mouse pancreas. Am J Physiol Gastrointest Liver Physiol 2010; 299;(2.);G303-10. | Article | PubMed Abstract | PubMed Full Text

36. Clevers $\mathrm{H}$ : The cancer stem cell: premises, promises and challenges. $\mathrm{Nat}$ Med 2011; 17;(3.);313-9. | Article | PubMed

37. Merlos-Suarez A, Barriga FM, Jung P, Iglesias M, Cespedes MV, Rossell $D$, et al.: The intestinal stem cell signature identifies colorectal cancer stem cells and predicts disease relapse. Cell Stem Cell 2011; 8;(5.);51124. | Article | PubMed

38. Todaro M, Francipane MG, Medema JP, Stassi G: Colon cancer stem cells: promise of targeted therapy. Gastroenterology 2010; 138;(6.);2151-62. | Article | PubMed
39. Jin G, Ramanathan V, Quante M, Baik GH, Yang X, Wang SS, et al.: Inactivating cholecystokinin-2 receptor inhibits progastrin-dependent colonic crypt fission, proliferation, and colorectal cancer in mice. J Clin Invest 2009; 119;(9.);2691-701. | PubMed Abstract | PubMed Full Text

40. Sarkar S, Swiercz R, Kantara C, Hajjar KA, Singh P: Annexin A2 mediates up-regulation of NF-kappaB, beta-catenin, and stem cell in response to progastrin in mice and HEK-293 cells. Gastroenterology 2011; 140;(2.);583-95 e4. | Article | PubMed Abstract | PubMed Full Text

41. Bishnupuri KS, Luo Q, Sainathan SK, Kikuchi K, Sureban SM, Sabarinathan $M$, et al.: Reg IV regulates normal intestinal and colorectal cancer cell susceptibility to radiation-induced apoptosis. Gastroenterology 2010; 138;(2.);616-26, 26 e1-2. | Article | PubMed Abstract | PubMed Full Text

42. Quante M, Bhagat G, Abrams JA, Marache F, Good P, Lee MD, et al.: Bile acid and inflammation activate gastric cardia stem cells in a mouse model of Barrett-like metaplasia. Cancer Cell 2012; 21;(1.);36-51. | Article | PubMed

43. Tu SP, Quante M, Bhagat G, Takaishi S, Cui G, Yang XD, et al.: IFN-gamma inhibits gastric carcinogenesis by inducing epithelial cell autophagy and T-cell apoptosis. Cancer Res 2011; 71;(12.);4247-59. | Article I PubMed

44. Sureban SM, May R, Lightfoot SA, Hoskins AB, Lerner M, Brackett DJ, et al.: DCAMKL-1 regulates epithelial-mesenchymal transition in human pancreatic cells through a miR-200a-dependent mechanism. Cancer Res 2011; 71;(6.);2328-38. | Article | PubMed Abstract | PubMed Full Text

45. Sureban SM, May R, Ramalingam S, Subramaniam D, Natarajan G, Anant $S$, et al.: Selective blockade of DCAMKL-1 results in tumor growth arrest by a Let-7a MicroRNA-dependent mechanism. Gastroenterology 2009; 137;(2.);649-59, 59 e1-2. | Article | PubMed Abstract | PubMed Full Text

46. Sureban SM, May R, Mondalek FG, Qu D, Ponnurangam S, Pantazis $P$, et al:: Nanoparticle-based delivery of siDCAMKL-1 increases microRNA-144 and inhibits colorectal cancer tumor growth via a Notch-1 dependent mechanism. J Nanobiotechnology 2011; 9;(40. | Article | PubMed Abstract | PubMed Full Text

47. Ali N, Allam H, May R, Sureban SM, Bronze MS, Bader T, et al.: Hepatitis $\mathrm{C}$ virus-induced cancer stem cell-like signatures in cell culture and murine tumor xenografts. J Virol 2011; 85;(23.);12292-303. | Article | PubMed

48. Gagliardi G, Goswami M, Passera R, Bellows CF: DCLK1 immunoreactivity in colorectal neoplasia. Clin Exp Gastroenterol 2012; 5;(35-42. | Article | PubMed Abstract | PubMed Full Text

49. Vega KJ, May R, Sureban SM, Lightfoot SA, Qu D, Reed A, et al.: Identification of the putative intestinal stem cell marker doublecortin and CaM kinase-like-1 in Barrett's esophagus and esophageal adenocarcinoma. J Gastroenterol Hepatol 2012; 27;(4.);773-80. | Article I PubMed

\section{Citation:}

Gagliardi G and Bellows CF: DCLK1 expression in gastrointestinal stem cells and neoplasia. journal of Cancer Therapeutics and Research 2012, 1:12. http://dx.doi.org/10.7243/2049-7962-1-12 